\title{
Six-channel polychromator design and implementation for the UPC elastic/Raman LIDAR
}

\author{
Dhiraj Kumar, $^{1, *}$, Francesc Rocadenbosch ${ }^{1}$, Michaël Sicard ${ }^{1}$, Adolfo Comerón ${ }^{1}$, Constantino \\ Muñoz ${ }^{1}$, Diego Lange ${ }^{1}$, Sergio Tomás ${ }^{1}$, Eduard Gregorio ${ }^{2}$ \\ ${ }^{1}$ Dept. of Signal Theory and Communications (TSC), Remote Sensing Lab. (RSLAB), Universitat \\ Politècnica de Catalunya (UPC) / Institut d'Estudi Espacials de Catalunya (IEEC/CRAE). C/Jordi \\ Girona 1-3, E-08034, Barcelona, Spain.
}

${ }^{2}$ Department of Agroforestry Engineering, Universitat de Lleida (UdL), Lleida, Spain.

\begin{abstract}
A 6-channel dichroic-based polychromator is presented as the spectrally selective unit for the U.P.C. elastic/Raman lidar. Light emission is made at 355-nm (ultraviolet, UV), 532-nm (visible, VIS) and 1064-nm (near infrared, NIR) wavelengths. In reception, the polychromator is the spectral separation unit that separates the laser backscattered composite return into 3 elastic (355, 532, 1064-nm wavelengths) and 3 Raman channels (386.7, 607.4 and 407.5-nm (water-vapor) wavelengths). The polychromator houses photo-multiplier tubes (PMT) for all the channels except for the NIR one, which is avalanche photodiode (APD) based.

The optomechanical design uses 1-inch optics and Eurorack standards. The APD-based receiver uses a XY-axis translation/elevation micro-positioning stage due to its comparatively small active area and motorised neutral density filters are used in all PMT-based channels to avoid detector saturation. The design has been specially optimized to provide homogeneous spatial light distribution onto the photodetectors and good mechanical repeatability. All channels are acquired in mixed analog and photon-counting mode using Licel ${ }^{\circledR}$ transient recorders, which are controlled by means of a user friendly LabVIEW $W^{T M}$ interface.

The paper focuses on the main polychromator optical design parameters, that is, light collimation trade-offs, end-to-end transmissivity, net channel responsivity, light distribution and spot size onto the photodetectors.

The polychromator along with the rest of the U.P.C. lidar system has successfully been tested during a recent lidar system intercomparison campaign carried out in Madrid (Spain) during Oct. 2010.
\end{abstract}

Keywords: lidar, polychromator, spectral separation, light spatial distribution, transmissivity.

\section{INTRODUCTION}

The need of a multi-spectral lidar has widely been experienced in last few years with a view to invert the optical and microphysical properties of aerosols and their impact on the climate change. Henceforth and as a part of the EARLINETGALION objectives [1], a joint effort has already been made by the European Aerosol Research Lidar Network (EARLINET), which includes more than 25 research teams, however, still lacking the standardization and optimization of lidar network instruments [1][2][3]. While the quantitative retrieval of the optical components of extinction $(\alpha)$, backscatter $(\beta)$ and lidar ratio (LR) at the emission wavelength requires the combination of an elastic-type channel and a Raman channel, the retrieval of microphysical parameters of aerosols requires a minimum of $3+2$ channels [4][5][6]. Thus, the envisaged lidar instrument must include $3+2$ multispectral elastic/Raman channels. Currently, only 8 of the 25 EARLINET stations offer these specifications. The adaptation and operation of the U.P.C. Barcelona $3+3$ multispectral elastic/Raman station [7][8][9][10], which includes a third Raman channel for the measurement of atmospheric water vapour, has contributed to this goal.

\footnotetext{
${ }^{*}$ Correspondence: Dhiraj Kumar, C/Jordi Girona 1-3, Office : D4-016, E-08034 Barcelona, Spain; Email : dhiraj@tsc.upc.edu; Phone: 34- 93-401-68-49; Fax: 34-93-401-72-32; WWW: http//www-tsc.upc.edu/rslab
}

\footnotetext{
Lidar Technologies, Techniques, and Measurements for Atmospheric Remote Sensing VII, edited by Upendra N. Singh, Gelsomina Pappalardo, Proc. of SPIE Vol. 8182, 81820W (c) 2011 SPIE · CCC code: 0277-786X/11/\$18 · doi: 10.1117/12.896305
} 
This paper describes the design and implementation of the 6-channel dichroic based polychromator. The paper is organized as follows: Sec. 2 provides an overview of the polychromator optical and mechanical design, Sec.3 focuses on the key design considerations and trade-offs, and finally, Sec.4 gives conclusion remarks.

\section{POLYCHROMATOR DESIGN OVERVIEW}

The polychromator is the spectrally selective unit which separates the backscattered composite light return into the various wavelengths of interest. The U.P.C. polychromator is dichroic based, features 1-inch optics and has been designed for 3 elastic $(355,532,1064 \mathrm{~nm})$ and 3 Raman (386.7, 607.4 and 407.5nm (water-vapour) channels. The receiving optics front-end is formed by five photomultiplier tubes (PMT) and one avalanche photodiode (APD) based receivers. Three PMTs are used for the three Raman channels (386.7 and $607.4 \mathrm{~nm}$ atmospheric $\mathrm{N}_{2}$-Raman shifts, and 407.5nm water-vapour Raman shift). The other two PMTs are for the 354.7 and $532.1 \mathrm{~nm}$ elastic channels. All PMT and APD (1064nm)-based channels are simultaneously recorded in analog and photon-counting modes by means of an array of 6 mixed analog/photon counting Licel ${ }^{\circledR}$ transient recorders (Table.1). A fiber bundle is provisionally used to convey the composite lidar return from the telescope focal plane to the input of the polychromator.

\subsection{Optical design layout}

The polychromator optical layout (Table.2) has been simulated using ZEMAX ${ }^{\circledR}$ ray tracing software [11] (Fig.1). The main polychromator optical components are the dichroic filters (D1-D4), the lenses (L2-L4(1,2)), the beam splitter (BS1), the mirrors (M11, M12) as well as the interference filters (I1-I6) [7] and the corresponding detector eye-piece lenses (E1-E2). D1 is a long-wave pass filter that separates the UV wavelengths from the rest of the composite lidar return spectrum. D2, D3 and D4 respectively separate the 1064-nm wavelength from the composite 1064/607.4/532.1$\mathrm{nm}$ wavelength at the D2 input, the 607.4-nm wavelength from the composite 607.4/532.1-nm wavelengths at the D3 input, and finally, the 354.7-nm from the UV composite 354.7/ 386.7/407.5-nm wavelengths at the D4 input.

At the end of the two main optical paths (D1-M11 for the VIS-NIR sub band and D4-M12 for the UV sub band), the mirror $\mathrm{M} 1(1,2)$ is used to reflect the signal onto the last detector of each branch $(532.1$ and $407.5 \mathrm{~nm}$ detectors, respectively). Given the impossibility to find a dichroic filter selective enough to separate the two closest UV wavelengths of 407.5 and $386.7 \mathrm{~nm}$, a UV beamsplitter (BS1) is used, instead.

The functionality of the lenses is as follows: L2 tends to collimate the incoming light beam from the fiber bundle (an extended light source), L4(1,2) corrects for the divergence of the VIS/NIR beam (D1-M11 optical path) and UV beam (D4-M12 optical path), and lenses L3 focus the already separated light beams onto each respective photodetector. The distance between L3 and the active area of each respective photodetector can be slightly adjusted for each channel. In the mechanical construction of each receiver, the focusing lenses L3 and their respective interference filters are assembled into aluminium cylinders screwed to the opto-electronic receiver bodies. Different antireflection coatings have been used in all polychromator's lenses depending on the spectrum band they are used for. That way, the transmissivity of the lenses can be assumed nearly ideal (better than 93\%). Motorised neutral density filters and motorised shutter diaphragms are included in front of interference filters I1-I6.

In order to assist the optical layout design three pairs of rays have been simulated in ZEMAX ${ }^{\circledR}$ CAD ray-tracing software, one pair coming out from the center and the other two pairs coming out from the diametral ends of the fiber bundle at the entrance of the polychromator (i.e. FB in Fig.1). This corresponds to a distance of $-1.5,0$ and $+1.5 \mathrm{~mm}$ ( $3 \mathrm{~mm}$ fiber size) from the optical axis. The angles of incidence of these rays were calculated from the numerical aperture of the fiber bundle. To reduce position sensitivity problems, the distance between the lenses L3 and their respective photodetectors was chosen so that the simulated spot sizes onto the detector active areas are approximately one half of their effective active areas. Uneven spatial responsivity effects over the detector active area are arranged out by means of the eye-piece pairs (E1 or E2) in all the channels. The optical set formed by the neutral density filter and the diaphragm has been placed between the focusing lens (L3) and the eye-piece (E1 or E2).

\subsection{Mechanical design layout}

The design goal at this stage has been to fit the polychromator unit into a light-sealed mechanical box following EURORACK dimension standards. The external mechanical box dimensions are $600 \mathrm{~mm}$ x $460 \mathrm{~mm} \times 150 \mathrm{~mm}(160 \mathrm{~mm}$ with its cover). The polychromator unit inside has an "L" shape with maximum dimensions $580 \mathrm{~mm} \times 180 \mathrm{~mm} \times 95 \mathrm{~mm}$. 
The complete mechanical drawing of the polychromator using computer aided design (AutoCAD ${ }^{\circledR}$ ) is shown in Fig.2. Because of its comparatively small active area, the APD-based receiver uses a XY-axis translation/elevation micropositioning stage (not shown in the figure). The optical axes of the polychromator are kept at $35 \mathrm{~mm}$ from the basis.

\section{DESIGN CONSIDERATIONS}

Light collimation.- In the multichannel polychromator designed here (Fig.1), the lens L2 is used as a nearly collimating lens. Since the input light source to the polychromator (a fiber bundle, composed of $\sim 400$ fibers, (FB) in Fig. 1 ) is not a point source but an extended light source, perfect collimation (i.e. obtaining parallel light rays at the output of L2) is not possible. In contrast, L2 is moved slightly off-focus with respect to the fiber bundle in order to minimize the beam divergence at its output (L2-L4 (1,2) optical path). Based on Zemax ${ }^{\circledR}$ simulation the FB-L2 distance was determined to be $d=34 \mathrm{~mm}$ which corresponds to $d=0.90 f$, with $f$ the focal-length of L2. Therefore, L2 was located off-focus and closer to the fiber bundle.

Light divergence.- Following the methodology presented in the [ANNEX A], light divergence at the output of the collimating lens system (single lens L2 in the case of 355nm channel, L2-L4(1,2) lens pair for all other channels, Fig.1) can be assessed. This divergence $(\sim 28 \mathrm{mrad})$ is less than one fourth the numerical aperture of the bundle.

Assessing the maximum beam divergence of the "collimated" beam is important for two reasons: (i) in order to estimate the centre wavelength (CWL) de-tuning of the interference filters and, consequently, their minimum required bandwidth and (ii) in order to specify the required eye-pieces to be placed in front of the photo-detectors (i.e. at the end of the each optical receiving path). The detector's eye-piece fulfils the important mission of averaging spatial responsivity inhomogeneties over the detector's active area.

Following [ANNEX B] and for a 28mrad input divergence, the maximum CWL shift is about $0.12 \mathrm{~nm}(1064 \mathrm{~nm}$, the worst channel) and the minimum bandwidth required is $\approx 0.24 \mathrm{~nm}(2 \times 0.12 \mathrm{~nm}$, the largest center wavelength shift occurring at $1064 \mathrm{~nm}$ ). In those conditions and given a $15 \%$-error bar for the CWL specified by the manufacturer, a minimum bandwidth of $0.3 \mathrm{~nm}$ is needed to guarantee reception, however, a more conservative figure of $1 \mathrm{~nm}$ is used, instead.

Spatial uniformity in the responsivity of the used photodetectors.- The image of the backscattered lidar return in the telescope focal plane always has 'comma' like shape with its head due to the near range signal and its tail due to the far range signal. The use of a PMT detector with a non-uniform spatial responsivity to detect lidar signals can cause artifacts when transducing the lidar signal into an electrical signal. Previous studies [12] show that the normalized spatial uniformity on the active area of an 8mm PMT diameter can vary from 0.2 to 2.8 times the average value defined for the central part of the detector. Practically, the useful diameter of the Hamamatsu 7400 PMT (nominally $8 \mathrm{~mm}$ ) is only about $5 \mathrm{~mm}$ - including mounting and adjustment tolerances. The eyepiece (E1 or E2) not only makes the detector rather insensitive to several misalignments (axial/radial shifts) but also averages out spatial responsivity inhomogeneities. Axial misalignments are the the most critical as it is difficult to determine the exact location of the PMT cathode with respect to the PMT housing [13]. As can be seen in the full-field spot diagrams simulated in Zemax ${ }^{\circledR}$ (Fig.3), insertion of eyepiece is very effective in terms of spreading the incoming rays onto the entire surface of the detectors [ANNEX C].

End-to-end transmissivity.- In order to achieve the highest total (or "net") channel responsivity and signal-to-noise ratio, it is necessary to minimize transmission losses due to all the optical components in the polychromator reception chain. At this point, selection of the best coatings in all the optical components used (antireflection coatings in dichroics and lenses) is essential. The end-to-end transmissivity of a channel is defined as

$$
T_{n e t}(\lambda)=T_{t}(\lambda) \cdot T_{b}(\lambda) \cdot T_{o p}(\lambda),
$$

where $T_{t}(\lambda)$ is the telescope transmission, $T_{b}(\lambda)$ is the fiber-bundle transmission and $T_{o p}(\lambda)$ is the polychromator transmission for the channel at wavelength $\lambda$.

Fig.4 shows the spectral response curve for the antireflection coatings used for dichroics D1 and D4 (spectral response better than 90\%). The end-to-end transmissivity for each channel is listed in Table.2.

\section{CONCLUSION}


A 6-channel polychromator for the elastic/Raman scanning lidar system at the Remote Sensing Lab (RSLab) of the U.P.C. Barcelona has been presented. The polychromator has been fully operational and successfully tested during recent EARLINET (European Aerosol Research Lidar Network) intercomparison campaign carried out in Madrid (Spain) in Oct. 2010. Main optical design considerations including light collimation trade-offs, interference filter bandwidth, endto-end transmissivity and light distribution onto the photodetectors have been discussed. A comprehensive optical design layout and details of its mechanical construction have been shown.

Future research work looks forward to achieving full validation of the multi-spectral lidar system in terms of simulated energy link-budget and experimental data results.

\section{ACKNOWLEDGEMENTS}

This work was supported by the European Union under the project ACTRIS (Aerosols, clouds, and Trace gases Research Infrastructure Network) ref. $n^{0}$ FP7- INFRA-2010-1.1.16, the European Space Agency under the project 21487/08/NL/HE, the Spanish Ministry of Science and Innovation (MICINN) and FEDER (European Regional Development) funds under the project TEC2009-09106 and Complementary Actions CGL2009-08031-E/CLI (“Charmex") and CGL2008-01330-E/CLI. Generalitat Catalunya /AGAUR is thanked for Mr. D. Kumar's pre-doctoral fellowship.

\section{REFERENCES}

[1]. Bösenberg, J. and Matthias, V., "EARLINET: A European Aerosol Research Lidar Network to Establish an Aerosol Climatology", Final report Feb. 2000-2003, Max-Planck Institut für Meteorologie, Hamburg (Germany), 191 (2003).

[2]. Behrendt, A., Nakamura, T., Onishi, M., Baumgart, R. and Tsuda, T., "Combined Raman lidar for the measurement of atmospheric temperature, water vapor, particle extinction coefficient, and particle backscatter coefficient," App. Opt. 41(36), 7657-7666 (2002).

[3]. Mattis, I., Ansmann, A., Althausen, D., Jaenisch, V., Wandinger, U., Muller, D., Arshinov, Y., F., Bobrovnikov, S. M. and Serikov, I. B., "Relative-humidity profiling in the troposphere with a Raman lidar," App. Opt. 41(30), 6451-6462 (2002).

[4]. Ansmann, A., Wandinger, U., Riebesell, M., Weitkamp, C. and Michaelis, W., "Independent measurement of extinction and backscatter profiles in cirrus clouds by using a combined Raman elastic-backscatter lidar," App. Opt. 31(33), 7113-7131 (1992).

[5]. Goldsmith, J., E., M., Blair, F., H., Bisson, S., E. and Turner D., D., "Turn-key Raman lidar for profiling atmospheric water vapor, clouds, and aerosols," App. Opt. 37(21), 4979-4990 (1998).

[6]. Böckmann, C., Müller, D., Osterloh, L., Pornsawad, P. and Papayannis, A., "From EARLINET ASOS RamanLidar signals to microphysical aerosol properties via advances regularizing software," Proc. IEEE International Geoscience and Remote Sensing Symposium, (II-422)-(II-425) (2008).

[7]. Kumar, D., Sicard, M., Tomas, S., Munoz, C., Rocadenbosch, F. and Comeron, A., "Engineering of a water vapour, Raman, elastic-backscatter Lidar at the Technical University of Catalonia (Spain)," Proc. SPIE, Vol. 6367, 63670U/1-63670U/12 (2006).

[8]. Sicard, M., Reba, M. N. Md, Rocadenbosch, F., Gregorio, E., Kumar, D., Tomás, S., Comeron, A., Molero, F., Pujadas, M., Guerrero-Rascado, J. L., Alados-Arboledas, L., Pedrós, R. and Martínez, J. A., "Intercomparison of Spanish advanced lidars in the framework of EARLINET," Proc. IEEE International Geoscience and Remote Sensing Symposium, 2763-2766 (2007).

[9]. Rocadenbosch, F., "Lidar-Aerosol Sensing," in Encyclopedia of Optical Engineering, D. D. Driggers, Ed., (Marcel Dekker, New York, 2003), pp. 1090-1102. ISSN 0.8247-0939-X (print format), ISSN 0-8247-0940-3 (electronic format).

[10]. Rocadenbosch, F., "Lidar-Wind, Raman and Other Sensing," in Encyclopedia of Optical Engineering, Donald D. Driggers, Ed., (Marcel Dekker, New York, 2003), pp. 1114-1127. ISSN 0.8247-0939-X (print format), ISSN 0-8247-0940-3 (electronic format).

[11]. http://www.zemax.com (Zemax ${ }^{\circledR}$ manual, accessed on Jul.2011)

[12]. Simeonov, V., Larcheveque, G., Quaglia, P., Bergh, H. and Calpini, B., "Influence of the photomultiplier tube spatial uniformity on lidar signals," App. Opt. 38(24), 5186-5190 (1999). 
[13]. Freudenthaler, V., "Effects of spatially inhomogeneous photomultiplier sensitivity on lidar signals and remedies", Proc. International Laser Radar Conference 22, 37-40 (2004).

[14]. Möller, K.D., "Geometrical Optics," in Optics: Learning by Computing with example using Mathcad ${ }^{\mathbb{B}}$, Matlab $^{\circledR}$, Mathematica ${ }^{\circledR}$ and Maple ${ }^{\circledR}$ (Springer Science+Business Media, New York, 2007), pp. 1-73, ISBN 13: 978-0-387-26168-3 (print format), ISBN 13: 978-0-387-69492-4 (electronic format).

[15]. www.omegafilters.com/index.php?page=tech_fd_ap (accessed on Jul.2011)

\section{FIGURES AND TABLES}
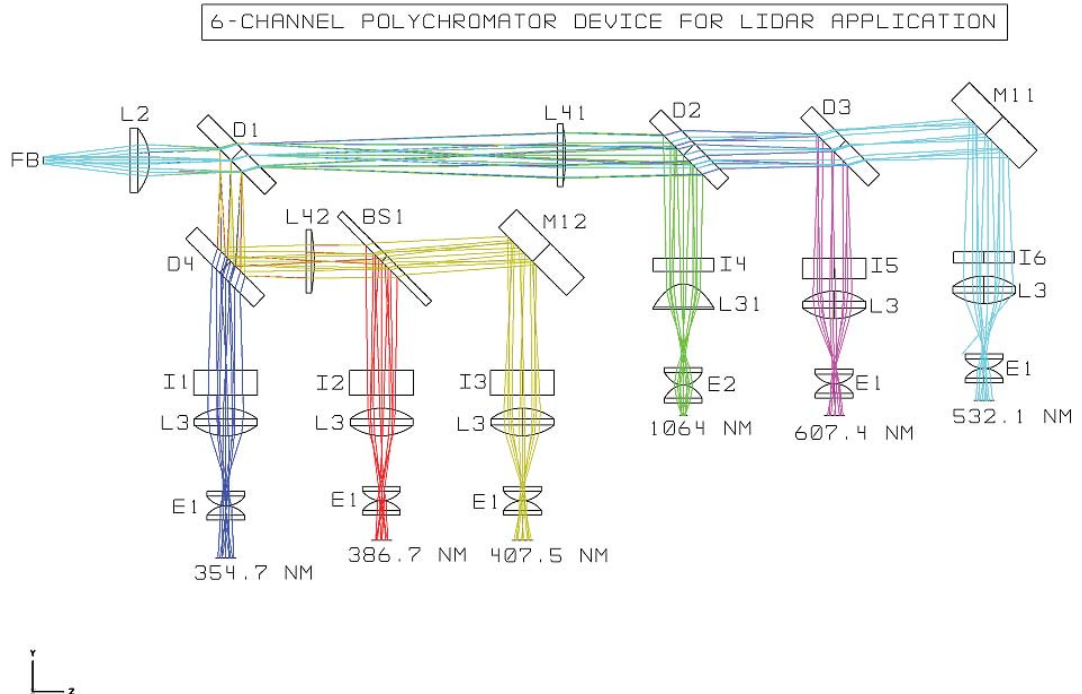

Fig.1. 3+3 Polychromator optical layout and related ZEMAX ${ }^{\circledR}$ ray tracing at $354.7 \mathrm{~nm}, 532.1 \mathrm{~nm}$, and $1064 \mathrm{~nm}$ (elastic channels), $386.7 \mathrm{~nm}$ and $607.4 \mathrm{~nm}\left(\mathrm{~N}_{2}\right.$ Raman channels), and $407.5 \mathrm{~nm}$ (water vapour Raman channel). The fiber bundle is indicated by "FB", dichroic filters by "D", the lenses by "L", the mirrors by "M", the beamsplitters by "BS", and the interference filters by "I".
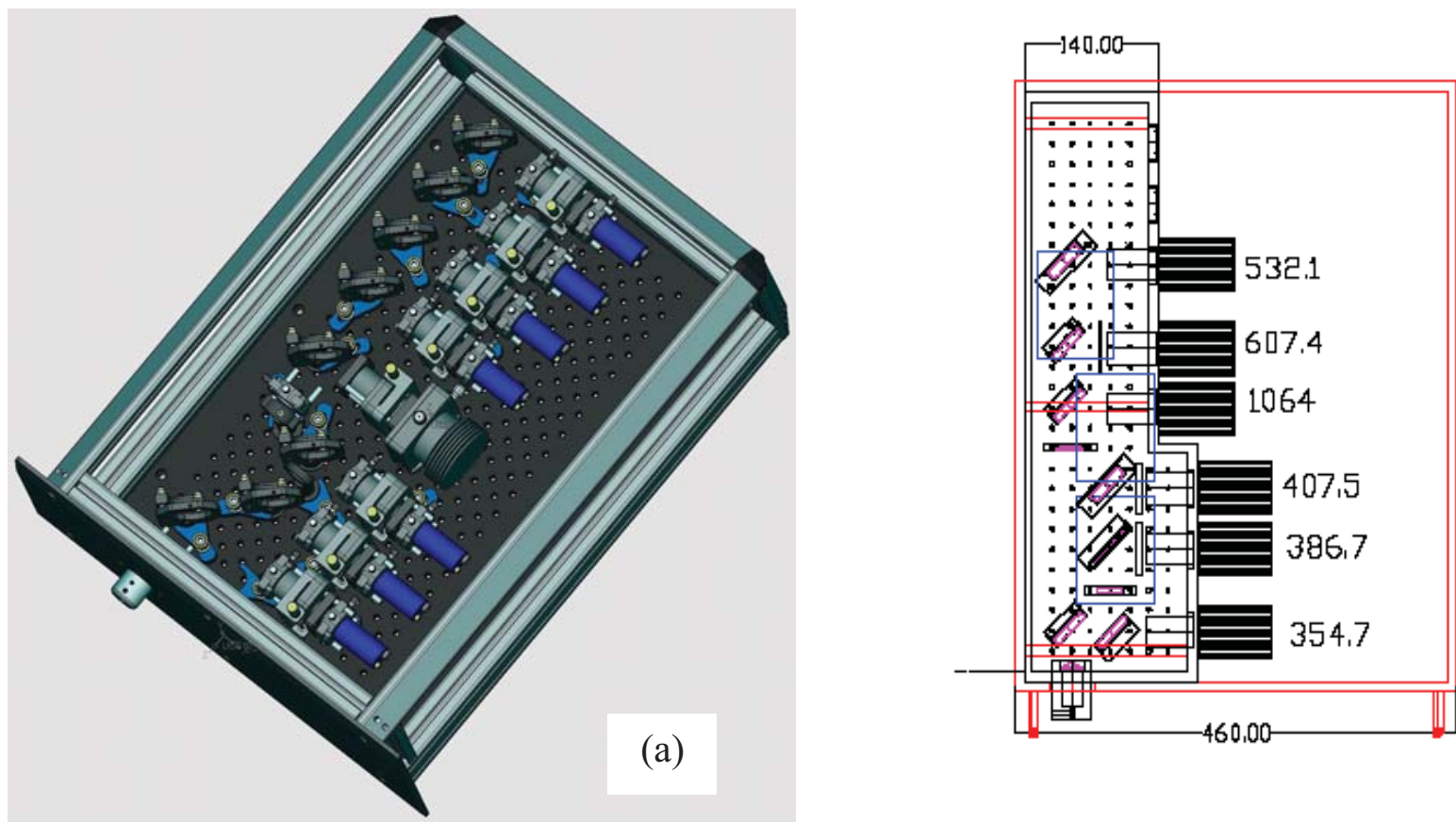

Fig.2. 3+3 Polychromator opto-mechanical implementation (AUTOCAD ${ }^{\circledR}$ ) (a) Rack-mounting implementation (also showing two future extra channels added (7 PMT-based and 1 APD-based)) (ii) Optical layout 


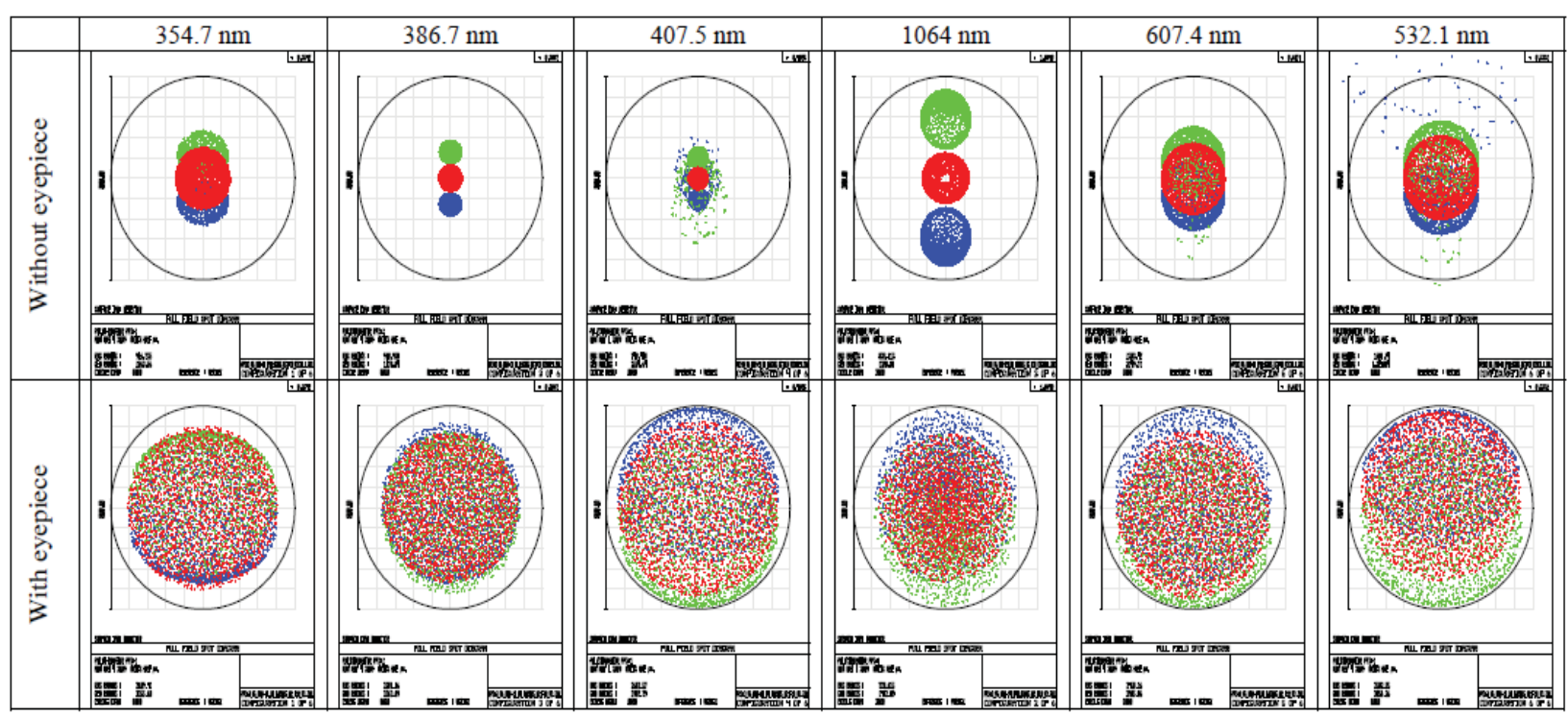

Fig.3. Effect of the eye-piece (L3 in Fig.C.1) on the light distribution over the detector's active area (Top) Without eye-piece (Bottom) With eye-piece (different colours correspond to rays emerging from different diametral positions along the bundle aperture (see FB in Fig.1)
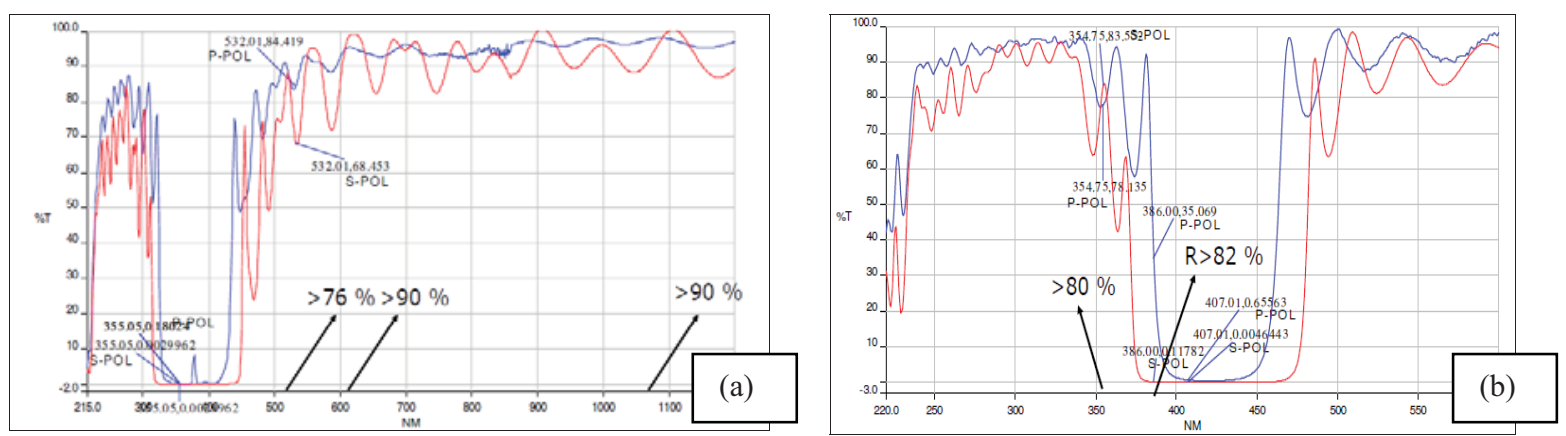

Fig.4. Dichroic coating spectral response (a) Coating response for dichroic D1 (b) Coating response for dichroic D4 (Table.2) 
Table.1: $3+3$ channel polychromator lidar system technical specifications

\begin{tabular}{|c|c|c|c|c|c|c|}
\hline \multicolumn{7}{|c|}{ EMITTER } \\
\hline Laser & \multicolumn{3}{|c|}{$\begin{array}{l}\text { Model / Type } \\
\text { Wavelengths } \\
\text { Energy per pulse } \\
\text { Repetition Rate / pulse duration } \\
\end{array}$} & \multicolumn{3}{|c|}{$\begin{array}{l}\text { Quantel Brilliant T64 / Nd:YAG Q-Switched } \\
1064 \text { / } 532 \text { / 355nm } \\
130 / 130 \text { / 40mJ } \\
20 \mathrm{~Hz} / 3.6 \mathrm{~ns} \\
\end{array}$} \\
\hline \multicolumn{7}{|c|}{ RECEIVER } \\
\hline Telescope & \multicolumn{3}{|c|}{$\begin{array}{l}\text { Model / Geometry } \\
\text { Primary / shadow diameter } \\
\text { Focal length }\end{array}$} & \multicolumn{3}{|c|}{$\begin{array}{l}\text { Celestron CGE } 1400 \text { / Schmidt-Cassegrain } \\
0.35 \mathrm{~m} / 0.11 \mathrm{~m} \\
3.91 \mathrm{~m}\end{array}$} \\
\hline Fiber Bundle & \multicolumn{3}{|c|}{$\begin{array}{l}\text { Numerical aperture }(\mathrm{NA}) \\
\text { Area diameter / Estimated transmission }\end{array}$} & \multicolumn{3}{|c|}{$\begin{array}{l}0.12 \\
3 \mathrm{~mm} / 50 \%\end{array}$} \\
\hline Polychromator & \multicolumn{6}{|c|}{ Ad hoc design } \\
\hline Photodetectors & \multicolumn{3}{|c|}{$\begin{array}{l}\text { Elastic channel }(1064 \mathrm{~nm}) \\
\text { Rest of channels } \\
\text { Active area diameter }\end{array}$} & \multicolumn{3}{|c|}{$\begin{array}{l}\text { PMT (Hamamatsu) } \\
\text { APD (3mm)/PMT (8mm) }\end{array}$} \\
\hline Channel Acquisition & \multicolumn{3}{|c|}{$\begin{array}{l}\text { Model } \\
\text { Type }\end{array}$} & \multicolumn{3}{|c|}{$\begin{array}{l}\text { Licel transient recorder TR40-80 } \\
\text { Mixed } 250 \mathrm{MHz} \text { PC + ADC 40Msps/12bit }\end{array}$} \\
\hline \multicolumn{7}{|c|}{ CHANNEL SPECIFICATIONS } \\
\hline Wavelength [nm] & 1064 & 532.1 & 354.7 & 386.7 & 607.4 & 407.5 \\
\hline Type & \multicolumn{3}{|c|}{ Elastic } & \multicolumn{2}{|l|}{ Raman $355 / \mathrm{N}_{2}$} & $355 / \mathrm{H}_{2} \mathrm{O}$ \\
\hline Spatial resolution $[\mathrm{m}]$ & \multicolumn{6}{|c|}{3.75} \\
\hline End-to-end transmissivity (\%) & 7.49 & 8.29 & 6.18 & 6.79 & 18.33 & 5.76 \\
\hline Spectral Bandwidth [nm] & 1 & 1 & 1 & 1 & 1 & 1 \\
\hline Detector type & APD & \multicolumn{5}{|c|}{ PMT } \\
\hline Detector model & $\mathrm{C} 30956 \mathrm{E}$ & R7400P-03 & R7400U-03 & R7400U-03 & R7400U-20 & R7400U-20 \\
\hline Internal Gain & 100 & $10^{6}$ & $7 \cdot 10^{5}$ & $7 \cdot 10^{5}$ & $5 \cdot 10^{5}$ & $5 \cdot 10^{5}$ \\
\hline Noise Factor & 4 & 1.3 & 1.3 & 1.3 & 1.3 & 1.3 \\
\hline Dark current $[\mathrm{nA}] \quad\left(25^{\circ} \mathrm{C}\right)$ & 89.2 & 0.08 (anode) & 0.01 (anode) & 0.01 (anode) & 0.64 (anode) & 0.86 (anode) \\
\hline Voltage Responsivity [V/W] & $3.7 \cdot 10^{5}$ & $1.5 \cdot 10^{6}$ & $1.9 \cdot 10^{6}$ & $2.1 \cdot 10^{6}$ & $2.0 \cdot 10^{6}$ & $7.5 \cdot 10^{5}$ \\
\hline System NEP $\left[\mathrm{fW} / \mathrm{Hz}^{-1 / 2}\right]$ & 2870 & 7.7 & 7.7 & 6.4 & 3.0 & 26.2 \\
\hline
\end{tabular}

(PMT stands for photomultiplier tube, APD for avalanche photo diode, PC for photon counting mode, ADC for analog-to-digital converter and NEP for Noise Equivalent Power). 
Table.2: Optical components specification

\begin{tabular}{|c|c|c|c|c|c|c|}
\hline \multicolumn{2}{|l|}{ Lenses } & \multicolumn{2}{|l|}{ L2 } & \multicolumn{2}{|l|}{ L3 } & L4(1,2) \\
\hline \multicolumn{2}{|l|}{ Type } & \multicolumn{2}{|c|}{ Plano-convex } & \multicolumn{2}{|l|}{ Bi-convex } & Plano-convex \\
\hline \multicolumn{2}{|c|}{ Effective focal length (mm) } & \multicolumn{2}{|l|}{38.0} & \multicolumn{2}{|l|}{25.4} & 150 \\
\hline \multicolumn{2}{|c|}{$\Phi(\mathrm{mm})$} & \multicolumn{2}{|l|}{25.4} & \multicolumn{2}{|l|}{25.4} & 25.4 \\
\hline Dichroics & \multicolumn{2}{|l|}{ D1 } & D2 & \multicolumn{2}{|l|}{ D3 } & D4 \\
\hline Reflectivity & \multicolumn{2}{|c|}{$\begin{array}{l}\geq 99 \% \text { at } 407,386, \\
355 \mathrm{~nm}\end{array}$} & $\geq 99 \%$ at $1064 \mathrm{~nm}$ & \multicolumn{2}{|c|}{$\geq 98 \%$ at $607 \mathrm{~nm}$} & $\geq 98 \%$ at $407 \mathrm{~nm}$, \\
\hline Transmissivity & \multicolumn{2}{|c|}{$\begin{array}{l}\geq 85 \% \text { at } 1064,607, \\
532 \mathrm{~nm}\end{array}$} & $\begin{array}{l}\geq 80 \% \text { at } 607, \\
532 \mathrm{~nm}\end{array}$ & \multicolumn{2}{|c|}{$\geq 60 \%$ at $532 \mathrm{~nm}$} & $\geq 60 \%$ at $355 \mathrm{~nm}$ \\
\hline Mirror & \multicolumn{3}{|l|}{ M1 $(1,2)$} & Beamsplitter & \multicolumn{2}{|l|}{$\mathrm{BS}$} \\
\hline Diameter & \multicolumn{3}{|l|}{$38.00 \mathrm{~mm}$} & \multirow{2}{*}{\begin{tabular}{|l|} 
Diameter \\
Wavelengths \\
\end{tabular}} & \multicolumn{2}{|l|}{$50 \mathrm{~mm}$} \\
\hline Wavelengths & \multicolumn{3}{|l|}{$400-800 \mathrm{~nm}$} & & \multicolumn{2}{|c|}{$350-450 \mathrm{~nm}$} \\
\hline Interference filters & I1 & \multicolumn{2}{|l|}{$\mathrm{I} 2$} & I4 & I5 & I6 \\
\hline CWL (nm) & $354.7 \pm 0.45$ & $386.7 \pm 0.45$ & $407.5 \pm 0.45$ & $1064 \pm 2$ & $607.4 \pm 0.45$ & $532.1 \pm 2$ \\
\hline FWHM (nm) & 1 & 1 & 1 & 1 & 1 & 1 \\
\hline $\mathrm{T}(\%)$ & $>50$ & $>50$ & $>50$ & $>45$ & $>60$ & $>50$ \\
\hline $\mathrm{n}^{*}$ & 2.16 & 2.16 & 2.10 & 1.87 & 1.91 & 1.97 \\
\hline Eye-pieces & $\begin{array}{l}\mathrm{f}=18 \mathrm{~mm}, \\
\mathrm{~d}=15 \mathrm{~mm}\end{array}$ & $\begin{array}{l}\mathrm{f}=18 \mathrm{~mm}, \\
\mathrm{~d}=15 \mathrm{~mm}\end{array}$ & $\begin{array}{l}\mathrm{f}=18 \mathrm{~mm}, \\
\mathrm{~d}=15 \mathrm{~mm}\end{array}$ & $\begin{array}{l}\mathrm{f}=11.25 \mathrm{~mm}, \\
\mathrm{~d}=15 \mathrm{~mm}\end{array}$ & $\begin{array}{l}\mathrm{f}=18 \mathrm{~mm}, \\
\mathrm{~d}=15 \mathrm{~mm}\end{array}$ & $\begin{array}{l}\mathrm{f}=18 \mathrm{~mm}, \\
\mathrm{~d}=15 \mathrm{~mm}\end{array}$ \\
\hline
\end{tabular}

( $\mathrm{T}$ stands for transmissivity, $\mathrm{n}^{*}$ for effective refractive index, CWL for 'Center Wavelength' and FWHM for full width at half maximum). 


\section{ANNEX A: Beam divergence at the output of the collimation lens system}

In Fig. 1 the collimation lens system output is formed by either one single lens (L2) in the case of the $355 \mathrm{~nm}$ channel or by the pair of lenses (L2, L4(1,2)), separated a distance equal to the travelling path between they two, in the case of all other channels. The collimation lens system (formed by one or more lenses) is represented by L2' in Fig.A.1.

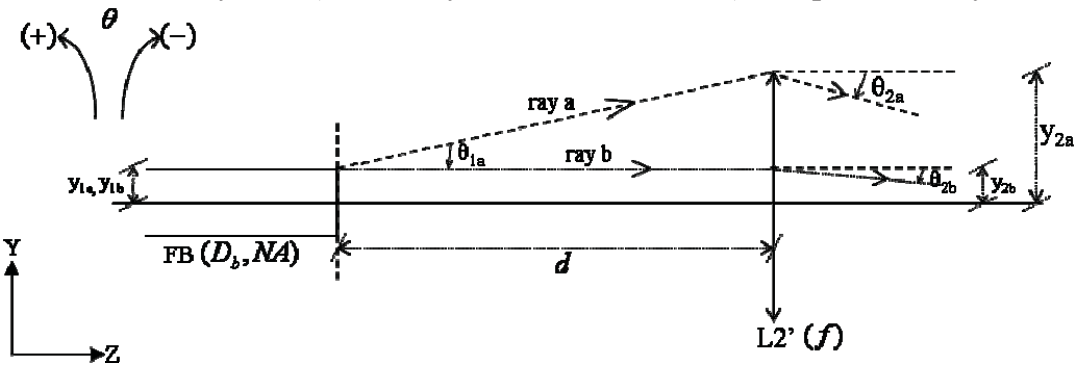

Fig.A.1. Calculation of light divergence inside the polychromator

In order to calculate the maximum beam divergence at the equivalent collimating lens output, we apply Abbe's invariant [14] to both rays $a$ and $b$ (Fig.A.1),

$$
\theta_{1 a} y_{1 b}-\theta_{1 b} y_{1 a}=\theta_{2 a} y_{2 b}-\theta_{2 b} y_{2 a}
$$

where the pairs $\left(\theta_{1 a}, y_{1 a}\right),\left(\theta_{1 b}, y_{1 b}\right)$ and $\left(\theta_{2 a}, y_{2 a}\right),\left(\theta_{2 b}, y_{2 b}\right)$ describe the corresponding angles and heights for the rays $a$ and $b$ at the fiber (FB) and the collimating lens (L2') outputs respectively. NA denotes the numerical aperture of the fiber, $D_{b}$ the fiber diameter and $f$ the equivalent focal-length of the collimating system (L2'). Using that $\left(\theta_{1 a}=\theta_{N A}, y_{1 a}=D_{b} / 2, \quad \theta_{1 b}=0, y_{1 b}=D_{b} / 2, \quad \theta_{2 b}=-\frac{D_{b} / 2}{f}, y_{2 b}=D_{b} / 2, \quad y_{2 a}=D_{b} / 2+\theta_{N A} \cdot d\right), \quad$ the light divergence at the output of the collimating lens system (L2') is given by

$$
\theta_{2 a}=N A-\frac{1}{f} \cdot\left(\frac{D_{b}}{2}+\theta_{N A} \cdot d\right)
$$

where $\theta_{N A}$ is defined as $\theta_{N A}=\sin ^{-1}(N A)$.

\section{ANNEX B: Filter's center wavelength de-tuning due to obliquitous incidence}

Optical filters such as the interference filters listed in Table.2 always specify their center wavelength (CWL) for normal incidence of rays at its input. When the input light beam has some divergence (A.2) or includes rays with obliquitous incidence $\left(\left[-\theta_{2 a}, \theta_{2 a}\right]\right)$, the CWL of the filter becomes shifted.

The CWL shift can be computed as [15]

$$
\lambda_{\theta}=\lambda_{0}\left[1-\left(\frac{n_{e}}{n^{*}}\right)^{2} \sin ^{2} \theta\right]^{\frac{1}{2}}
$$

where $\lambda_{\theta}$ is the CWL wavelength for obliquitous incidence, $\lambda_{0}$ is the CWL wavelength at normal incidence, $n_{e}$ is the refractive index of external medium ( $n_{e}=1$, air), $n^{*}$ is the effective refractive index of the filter and $\theta$ is the angle of incidence. Table (B.1) shows the CWL shift for the polychromator's channels in response to a maximum angle of incidence ( $\theta=\theta_{2 a} \cong 28 \mathrm{mrad}$, Eq.(A.2), An.A)

Table. B.1: Estimated CWL shift for various channels ( $\left.\theta_{2 a} \cong 28 \mathrm{mrad}\right)$

\begin{tabular}{|c|c|c|c|}
\hline$\lambda_{0}(\mathrm{~nm})$ & $n^{*}$ & $\lambda_{\theta}(\mathrm{nm})$ & $\Delta \lambda=\left|\lambda_{\theta}-\lambda_{0}\right|$ \\
\hline 386.7 & 2.16 & 386.67 & 0.03 \\
\hline 407.5 & 2.10 & 407.46 & 0.04 \\
\hline
\end{tabular}




\begin{tabular}{|c|c|c|c|}
\hline 607.4 & 1.91 & 607.33 & 0.07 \\
\hline 354.7 & 2.16 & 354.67 & 0.03 \\
\hline 532 & 1.97 & 531.95 & 0.05 \\
\hline 1064 & 1.87 & 1063.88 & $\mathbf{0 . 1 2}$ \\
\hline
\end{tabular}

\section{ANNEX C: Specification of the photo-detectors' eye-piece}

Fig.C.1 shows the equivalent optics layout of a polychromator channel. The fiber bundle (FB) conveys the backscattered light to the photodetector (D3) by combining the collimating lens (L1) [note that L1 stands for L2 (355nm channel) or the L2-L4(1,2) pair (all other channels) in Fig.1], the collecting lens (L2) and the eye-piece lens (L3). In order to specify the eye-piece lens in terms of size and focal-length, let's consider two rays leaving the fiber bundle at heights $+D_{b} / 2$ and $-D_{b} / 2$ and forming respective angles with the optical axis equal to NA of the bundle.

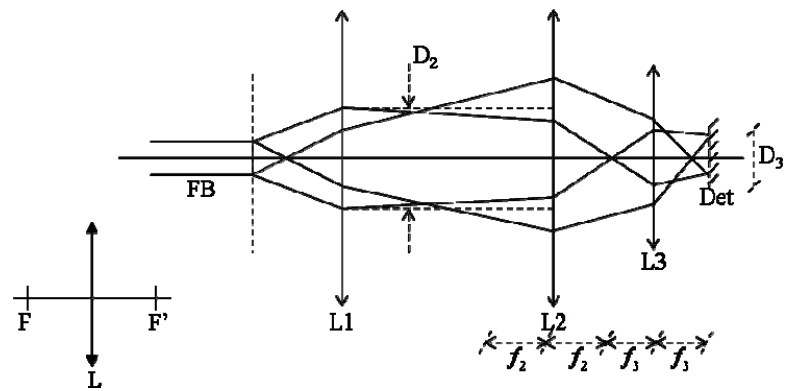

Fig.C.1. Equivalent optical layout of a polychromator channel. $\mathrm{D}_{2}$ stands for the maximum allowable diameter of the light beam in the L1-L2 path. $\mathrm{D}_{3}$ stands for the detector's active area diameter.

In order to average uneven spatial responsivity effects (Fig.3), the function of the lens L3 is to ensure a beam diameter of $8 \mathrm{~mm}$ (PMT) or $3 \mathrm{~mm}$ (APD) at the detector plane. This can be achieved when lenses L2 and L3 form an afocal system $\left(\mathrm{F} 2^{\prime}=\mathrm{F} 3\right)$ which means that parallel beams entering L2 will leave L3 also parallel. Therefore, the distance between the lenses L2 and L3 is $d=f_{2}+f_{3}$. With such a system, the beam diameters $D_{2}$ and $D_{3}$ are connected by paraxial approximation,

$$
\frac{D_{2}}{D_{3}}=\frac{f_{2}}{f_{3}} .
$$

Using standard 1-inch optics, assuming a maximum beam diameter $D_{2} \approx 20 \mathrm{~mm}$ (in any case $<25.4 \mathrm{~mm}$, the standard optics diameter), $f_{2}=25.4 \mathrm{~mm}$ (L3 in Table.2), $D_{3}=8 \mathrm{~mm}$ (PMT) or 3mm (APD), Eq.(C.1) yields $f_{3}=10.16 \mathrm{~mm}$ (PMT) and $f_{3}=3.81 \mathrm{~mm}(\mathrm{APD})$

Because typical f-numbers $(\# f=f / D$ ) are in the range $1.1 \ldots .4$ and the lens diameters are limited to $25.4 \mathrm{~mm}$, it was not possible to implement L3 specs with a single commercial lens. A combination of two plano-convex lenses (focallength $f$, equivalent focal-length $f_{\text {eq }}=\frac{f}{2}=f_{3}$ ) with the convex sides towards each other was used. 